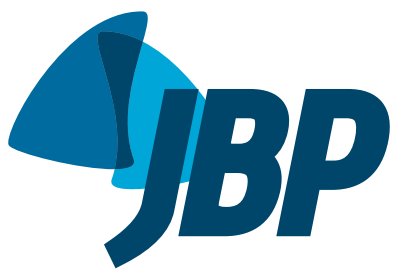

\title{
Resistance profile of strains of Mycobacterium fortuitum isolated from clinical specimens
}

\author{
Debora Ribeiro de Souza Santos ${ }^{1,2}$, Maria Cristina Silva Lourenço3, \\ Fábrice Santana Coelho ${ }^{4}$, Fernanda Carvalho Queiroz Mello ${ }^{5}$, \\ Rafael Silva Duarte ${ }^{6}$
}

\section{TO THE EDITOR:}

The Mycobacterium fortuitum group is associated with lung diseases in humans. This group is also responsible for most (60-80\%) cases of post-surgical and catheter-related infections caused by rapidly growing mycobacteria. ${ }^{(1)}$

In the present study, we evaluated 75 strains of the $M$. fortuitum group isolated from human clinical specimens, predominantly in the state of Rio de Janeiro, Brazil, and previously identified as $M$. fortuitum by hsp65 PCR-restriction enzyme analysis (PRA) in routine laboratories. ${ }^{(2,3)}$ These strains were isolated from patients between 2000 and 2010, various types of samples having been collected: sputum ( $n=49)$ from patients with respiratory symptoms, probable cases with clinical and radiological signs and one $M$. fortuitum isolation, $24 \%$ of the strains coming from confirmed cases of infection with more than one $M$. fortuitum isolation associated with the clinical and radiological profile; biopsies of nodules ( $n=$ $8)$; mammary secretions $(n=8)$, skin abscesses ( $n=$ $3)$, breast implant $(n=1)$, bronchial secretion $(n=1)$; bronchoalveolar lavage $(n=1)$; bone marrow aspirate $(n=1)$; urine $(n=2)$; and surgical wound secretion ( $n$ $=1$ ). Only 1 strain from each patient was included in the study. Antimicrobial susceptibility testing was performed as recommended by the Clinical and Laboratory Standards Institute. (4) Ofloxacin susceptibility was tested based on the study conducted by Wallace et al. ${ }^{(5)}$

There was significant variation among the 75 strains in terms of the in vitro response to the eight antimicrobial agents tested (Table 1 ). Approximately $86.6 \%$ of the strains of the $M$. fortuitum group $(n=65)$ exhibited susceptibility to amikacin with a minimum inhibitory concentration (MIC) of $1-16 \mu \mathrm{g} / \mathrm{mL}$. For cefoxitin, the proportion of resistant strains was quite high, $96 \%$ ( $n=72$ ), considering the categories "resistant" and "intermediate", with an MIC of 32-256 $\mu \mathrm{g} / \mathrm{mL}$. The same was observed for clarithromycin, to which the resistance rate was $94.6 \%(n=71)$, with an MIC of $8-32 \mu \mathrm{g} / \mathrm{mL}$. For the fluoroquinolone group, susceptibility rates and MIC values for ciprofloxacin, moxifloxacin, and ofloxacin were, respectively, $88 \%(n=66)$ and $\leq 1 \mu \mathrm{g} / \mathrm{mL} ; 94.6 \%$ $(\mathrm{n}=71)$ and $\leq 1 \mu \mathrm{g} / \mathrm{mL}$; and $78.6 \%(\mathrm{n}=59)$ and $\leq 2$ $\mu \mathrm{g} / \mathrm{mL}$. For doxycycline, we found a resistance rate of $68 \%(\mathrm{n}=51)$ and an MIC $\geq 1 \mu \mathrm{g} / \mathrm{mL}$. Trimethoprim/ sulfamethoxazole, in contrast with data in the literature, provided a resistance rate of $100 \%(n=75)$ with an MIC $\geq 4 / 76 \mu \mathrm{g} / \mathrm{mL}$ in all of the strains tested.

For each of the antimicrobial agents evaluated, we determined the MIC at which $50 \%$ of the isolates are inhibited $\left(\mathrm{MIC}_{50}\right)$, the MIC at which $90 \%$ of the isolates are inhibited $\left(\mathrm{MIC}_{90}\right)$, and the mode (Table 2).

It is of great importance to identify effective drug therapies for the various subspecies of the M. fortuitum group. ${ }^{(6)}$ In comparison with data in the literature on susceptibility profiles, some of our results were significantly different. According to one study, ${ }^{(1)}$ the $M$. fortuitum group exhibits susceptibility to the sulfonamides, represented by trimethoprim/sulfamethoxazole, $100 \%$ of the strains tested being sulfonamide-susceptible. In contrast, we observed a rate of resistance to trimethoprim/sulfamethoxazole of $100 \%$, with very high MIC values (> 8/152 $\mu \mathrm{g} / \mathrm{mL}$ ). Our results were obtained in strict accordance with the Clinical and Laboratory Standards Institute recommendations ${ }^{(4)}$ and underwent interlaboratory quality control assessment (data not shown).

According to a statement published by the American Thoracic Society/Infectious Disease Society of America, ${ }^{(7)}$ $80 \%$ of the $M$. fortuitum group is clarithromycin-susceptible and $50 \%$ of it is doxycycline-susceptible. However, despite the observed in vitro susceptibility, macrolides should be used with caution, because of the presence of the erythromycin-inducible methylase (erm) gene, which confers resistance to macrolides. ${ }^{(7,8)}$

One of the hypotheses that could explain the high rates of resistance to some antimicrobial agents in $M$. fortuitum is the widespread empiric use, in recent decades, of antibiotics for the treatment of nonspecific respiratory infections and of urinary tract infections, facilitated by patient access to these medications at no cost through the Brazilian Unified Health Care System and by convenient dosing schedules, which could exert a selective pressure on the samples. This is similar to what happens to $M$. tuberculosis strains following exposure to quinolones, as reported in two studies. ${ }^{(9,10)}$ According to the study conducted by Brown-Elliott et al., ${ }^{(1)}$ only rarely in cases of pretreatment with quinolones will strains of the $M$. fortuitum group be resistant to quinolones, including

1. Programa de Pós-Graduação em Clínica Médica, Hospital Universitário Clementino Fraga Filho, Universidade Federal do Rio de Janeiro, Rio de Janeiro (RJ) Brasil.

2. Departamento de Ciências Biológicas, Escola Nacional de Saúde Pública Sérgio Arouca, Fundação Oswaldo Cruz, Rio de Janeiro, Rio de Janeiro (RJ) Brasil.

3. Instituto Nacional de Infectologia Evandro Chagas, Fundação Oswaldo Cruz, Rio de Janeiro, Rio de Janeiro (RJ) Brasil.

4. Hospital Universitário Pedro Ernesto, Universidade do Estado do Rio de Janeiro, Rio de Janeiro (RJ) Brasil.

5. Instituto de Doenças do Tórax, Universidade Federal do Rio de Janeiro, Rio de Janeiro (RJ) Brasil

6. Instituto de Microbiologia Paulo de Góes, Universidade Federal do Rio de Janeiro, Rio de Janeiro (RJ) Brasil. 
Table 1. Minimum inhibitory concentration of antimicrobial agents for the 75 strains of the Mycobacterium fortuitum group.

\begin{tabular}{|c|c|c|c|c|c|c|c|c|c|c|c|c|}
\hline \multirow[t]{2}{*}{ Antimicrobial agent } & \multicolumn{12}{|c|}{ MIC, $\mu \mathrm{g} / \mathrm{mL}$} \\
\hline & 256 & 128 & 64 & 32 & 16 & 8 & 4 & 2 & 1 & 0.5 & 0.25 & 0.125 \\
\hline Amikacin & & 7 & & 3 & 5 & 10 & 15 & 24 & 11 & & & \\
\hline Cefoxitin & 16 & 19 & 18 & 19 & 1 & & & 2 & & & & 5 \\
\hline Ciprofloxacin & & & & & 7 & 1 & & 1 & 14 & 17 & 6 & 29 \\
\hline Clarithromycin & & & 1 & 2 & 42 & 26 & 1 & & & 3 & & 6 \\
\hline Doxycycline & & & & 42 & 1 & 5 & & 3 & & 2 & 22 & \\
\hline Moxifloxacin & & & & & 2 & 1 & 1 & & 47 & 1 & 1 & 22 \\
\hline \multirow[t]{3}{*}{ Ofloxacin } & & & & & & & 16 & 9 & 12 & 11 & 17 & 10 \\
\hline & \multicolumn{12}{|c|}{ MIC, $\mu \mathrm{g} / \mathrm{mL}$} \\
\hline & \multicolumn{2}{|c|}{$16 / 304$} & \multicolumn{2}{|c|}{$8 / 152$} & \multicolumn{2}{|c|}{$4 / 76$} & \multicolumn{2}{|c|}{$2 / 38$} & \multicolumn{2}{|c|}{$1 / 19$} & \multicolumn{2}{|c|}{$0.5 / 9.5$} \\
\hline $\begin{array}{l}\text { Trimethoprim/ } \\
\text { sulfamethoxazole }\end{array}$ & \multicolumn{2}{|c|}{66} & \multicolumn{2}{|c|}{8} & \multicolumn{2}{|c|}{1} & & & & & & \\
\hline
\end{tabular}

MIC: minimum inhibitory concentration.

Table 2. Minimum inhibitory concentration (at which $50 \%$ and $90 \%$ of the isolates are inhibited) of antimicrobial agents for the 75 strains of the Mycobacterium fortuitum group.

\begin{tabular}{lccccc}
\multicolumn{1}{c}{ Antimicrobial agent } & Range & Mode & MIC $_{50}, \boldsymbol{\mu} \mathbf{g} / \mathbf{m L}$ & $\mathbf{M I C}_{90}, \boldsymbol{\mu} \mathbf{g} / \mathbf{m L}$ & Susceptibility, \% \\
Amikacin & $128-1$ & 2 & 4 & $<32$ & 86.6 \\
Cefoxitin & $256-2$ & 64 & 64 & 256 & 4.0 \\
Ciprofloxacin & $16-0.125$ & 0.5 & 0.5 & 4 & 88.0 \\
Clarithromycin & $64-0.5$ & 16 & 16 & 16 & 5.4 \\
Doxycycline & $32-0.25$ & 32 & 32 & 32 & 32.0 \\
Moxifloxacin & $16-0.125$ & 1 & 1 & 16 & 94.6 \\
Ofloxacin & $4-0.125$ & 4 & 1 & 4 & 78.6 \\
Trimethoprim/sulfamethoxazole & $16 / 304-0.5 / 9.5$ & $16 / 304$ & $16 / 304$ & $16 / 304$ & 0.0 \\
\hline
\end{tabular}

MIC: minimum inhibitory concentration; $\mathrm{MIC}_{50}$ : MIC at which $50 \%$ of the isolates are inhibited; and $\mathrm{MIC}_{90}$ : $\mathrm{MIC}$ at which $90 \%$ of the isolates are inhibited.

ciprofloxacin and moxifloxacin. Such exposure could explain the identification of some quinolone-resistant isolates.

The role of empiric fluoroquinolone therapy for community-acquired pneumonia remains controversial in countries with a high incidence of tuberculosis, because of the possibility of delay in the diagnosis and treatment of tuberculosis, as well as of emergence of fluoroquinolone-resistant strains of $M$. tuberculosis. ${ }^{(10)}$ According to Singh, ${ }^{(9)}$ the guidelines on the management of community-acquired pneumonia in adults published by Mandell et al.(11) are very useful in developed countries, where the prevalence of tuberculosis is very low; however, they should not be applied in developing countries where the rate of tuberculosis is high. The guideline recommendations advocate the use of novel fluoroquinolones, such as gemifloxacin, levofloxacin, or moxifloxacin, to treat almost all categories of patients with community-acquired pneumonia. Because fluoroquinolones are broad-spectrum antimicrobial agents, their widespread, indiscriminate use, especially at subtherapeutic doses, is likely to increase quinolone resistance in microorganisms, including nontuberculous mycobacteria. ${ }^{(9)}$

In the present study, we found high resistance to quinolones and full resistance to trimethoprim/ sulfamethoxazole in the strains evaluated, the rates being significantly different from those reported previously. ${ }^{(1,7,8)}$ These data indicate the need to perform broth microdilution testing to determine susceptibility to antimicrobial agents and the need to enable the implementation of this method in the routine workflow of mycobacteriology laboratories, so that an effective and appropriate therapeutic approach can be developed.

\section{REFERENCES}

1. Brown-Elliott BA, Nash KA, Wallace RJ Jr. Antimicrobial susceptibility testing, drug resistance mechanisms, and therapy of infections with nontuberculous mycobacteria. Clin Microbiol Rev. 2012;25(3):545 82. http://dx.doi.org/10.1128/CMR.05030-11

2. Ringuet $H$, Akoua-Koffi $C$, Honore $S$, Varnerot A, Vicent V, Berche $P$, et al. hsp65 sequencing for identification of rapidly growing mycobacteria. J Clin Microbiol. 1999;37(3):852-7.

3. Telenti A, Marchesi F, Balz M, Bally F, Böttger EC, Bodmer T. Rapid identification of mycobacteria to the species level by polymerase chain reaction and restriction enzyme analysis. J Clin Microbiol. 1993;31(2):175-8.

4. Clinical and Laboratory Standards Institute (CLSI). Susceptibility Testing of Mycobacteria, Nocardiae, and Other Aerobic Actinomycetes; Approved Standard-Second Edition. Wayne (PA): CLSI; 2011 Mar. Standard No. M24-A2. 2011;31(5).

5. Wallace RJ Jr, Silcox VA, Tsukamura M, Brown BA, Kilburn JO Butler WR, et al. Clinical significance, biochemical features, and susceptibility patterns of sporadic isolates of the Mycobacterium 
chelonae-like organism J Clin Microbiol. 1993;31(12):3231-9.

6. Kirschner P, Kiekenbeck M, Meissner D, Wolters J, Böttger EC Genetic heterogeneity within Mycobacterium fortuitum complex species: genotypic criteria for identification. J Clin Microbiol. 1992;30(11):2772-5

7. Griffith DE, Aksamit T, Brown-Elliott BA, Catanzaro A, Daley C, Gordin $F$, et al. An official ATS/IDSA statement: diagnosis, treatment, and prevention of nontuberculous mycobacterial diseases. Am J Respir Crit Care Med. 2007;175(4):367-416. http://dx.doi.org/10.1164/ rccm.200604-571ST

8. Daley $\mathrm{CL}$, Griffith DE. Pulmonary non-tuberculous mycobacterial infections. Int J Tuberc Lung Dis. 2010;14(6):665-71.

9. Singh A. Fluoroquinolones should not be the first-line antibiotics to treat community-acquired pneumonia in areas of tuberculosis endemicity. Clin Infect Dis. 2007;45(1):133; author reply 134-5. http:// dx.doi.org/10.1086/518702

10. Shen GH, Tsao TC, Kao SJ, Lee JJ, Chen YH, Hsteh WC, et al. Does empirical treatment of community-acquired pneumonia with fluoroquinolones delay tuberculosis treatment and result in fluoroquinolone resistance in Mycobacterium tuberculosis? Controversies and solutions. Int J Antimicrob Agents. 2012;39(3):201 5. http://dx.doi.org/10.1016/.ijantimicag.2011.11.014

11. Mandell LA, Wunderink RG, Anzueto A, Bartlett JG, Campbell GD Dean NC, et al. Infectious Diseases Society of America/American Thoracic Society consensus guidelines on the management of community-acquired pneumonia in adults. Clin Infect Dis. 2007;44 Suppl 2:S27-72 http://dx.doi.org/10.1086/511159 